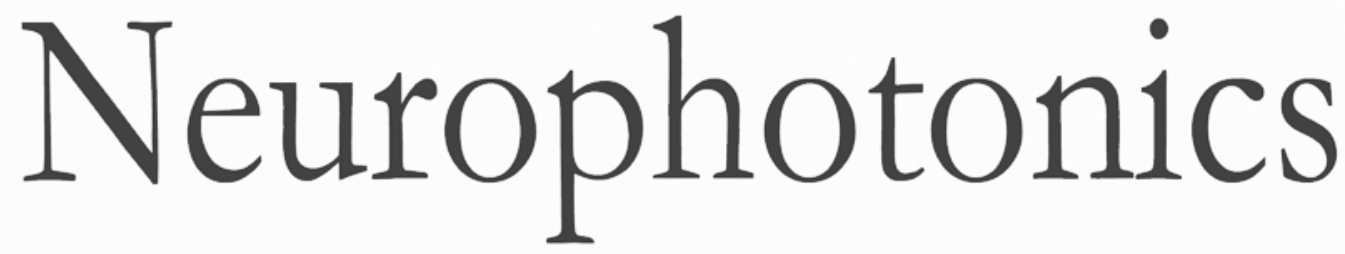

\title{
BabyLux device: a diffuse optical system integrating diffuse correlation spectroscopy and time-resolved near- infrared spectroscopy for the neuromonitoring of the premature newborn brain
}

Martina Giovannella

Davide Contini

Marco Pagliazzi

Antonio Pifferi

Lorenzo Spinelli

Rainer Erdmann

Roger Donat

Ignacio Rocchetti

Matthias Rehberger

Niels König

Robert Schmitt

Alessandro Torricelli

Turgut Durduran

Udo M. Weigel 


\title{
BabyLux device: a diffuse optical system integrating diffuse correlation spectroscopy and time-resolved near-infrared spectroscopy for the neuromonitoring of the premature newborn brain
}

\author{
Martina Giovannella, ${ }^{a}$ Davide Contini, ${ }^{b}$ Marco Pagliazzi, ${ }^{a}$ Antonio Pifferi, ${ }^{b, c}$ Lorenzo Spinelli, ${ }^{c}$ Rainer Erdmann, ${ }^{d}$ \\ Roger Donat, ${ }^{e}$ Ignacio Rocchetti, ${ }^{e}$ Matthias Rehberger, ${ }^{f}$ Niels König, ${ }^{f}$ Robert Schmitt, ${ }^{f, g}$ Alessandro Torricelli, ${ }^{b, c}$ \\ Turgut Durduran, ${ }^{a, h, \star}$ and Udo M. Weigel ${ }^{i}$ \\ aICFO-Institut de Ciències Fotòniques, The Barcelona Institute of Science and Technology, Castelldefels (Barcelona), Barcelona, Spain \\ ${ }^{\mathrm{b}}$ Politecnio di Milano-Dipartimento di Fisica, Milan, Italy \\ IIstituto di Fotonica e Nanotecnologie, Consiglio Nazionale delle Ricerche, Milan, Italy \\ dPicoQuant GmbH, Berlin, Germany \\ eLoop-Competitive Design Network, Sant Cugat del Vallès (Barcelona), Barcelona, Spain \\ ${ }^{f}$ Fraunhofer Institute for Production Technology IPT, Aachen, Germany \\ gRWTH Aachen University, Laboratory for Machine Tools and Production Engineering (WZL), Aachen, Germany \\ hInstitució Catalana de Recerca i Estudis Avançats, Barcelona, Spain. \\ 'HemoPhotonics S.L., Castelldefels (Barcelona), Barcelona, Spain
}

\begin{abstract}
The BabyLux device is a hybrid diffuse optical neuromonitor that has been developed and built to be employed in neonatal intensive care unit for the noninvasive, cot-side monitoring of microvascular cerebral blood flow and blood oxygenation. It integrates time-resolved near-infrared and diffuse correlation spectroscopies in a user-friendly device as a prototype for a future medical grade device. We present a thorough characterization of the device performance using test measurements in laboratory settings. Tests on solid phantoms report an accuracy of optical property estimation of about $10 \%$, which is expected when using the photon diffusion equation as the model. The measurement of the optical and dynamic properties is stable during several hours of measurements within $3 \%$ of the average value. In addition, these measurements are repeatable between different days of measurement, showing a maximal variation of $5 \%$ in the optical properties and $8 \%$ for the particle diffusion coefficient on a liquid phantom. The variability over test/retest evaluation is $<3 \%$. The integration of the two modalities is robust and without any cross talk between the two. We also perform in vivo measurements on the adult forearm during arterial cuff occlusion to show that the device can measure a wide range of tissue hemodynamic parameters. We suggest that this platform can form the basis of the next-generation neonatal neuromonitors to be developed for extensive, multicenter clinical testing. ๑ The Authors. Published by SPIE under a Creative Commons Attribution 4.0 Unported License. Distribution or reproduction of this work in whole or in part requires full attribution of the original publication, including its DOI. [DOI: 10.1117/1.NPh.6.2.025007]

Keywords: preterm infants; diffuse correlation spectroscopy; time-resolved near-infrared spectroscopy; neuromonitoring

Paper 19003R received Jan. 8, 2019; accepted for publication Apr. 12, 2019; published online May 10, 2019.
\end{abstract}

\section{Introduction}

Preterm birth is a major and widespread healthcare issue involving 15 million babies every year. ${ }^{1}$ It is a major cause of death in children under the age of 5 , and a high percentage of the survivors suffer neurological disabilities. ${ }^{2}$ The neurodevelopment of preterm newborns can be negatively affected by hemodynamic instabilities during the first hours and days of life, where brain lesions are often developed. ${ }^{3,4}$ It is hypothesized that brain-oriented intensive care of the preterm newborns would minimize these problems, but, to be effective, it would require the possibility to monitor the cerebral well-being at the cot-side in real time. Unfortunately, such a technology is still lacking in the daily clinical care.

A potential candidate is the continuous-wave near-infrared spectroscopy (CW-NIRS), ${ }^{5-7}$ which has already been commercialized in its spatially resolved spectroscopic implementation. ${ }^{8}$ These devices evaluate the regional cerebral microvascular

*Address all correspondence to Turgut Durduran, E-mail: turgut.durduran@ icfo.eu blood oxygenation and are relatively simple, inexpensive, noninvasive, and portable. ${ }^{9}$ CW-NIRS uses near-infrared light, because it is poorly absorbed by the tissue and is multiply scattered. This allows a deep penetration $(>1 \mathrm{~cm})$ of the light under the surface and its emergence on the same side of the tissue allowing for measurements on the forehead of the infant. ${ }^{10}$ Apart from this deep penetration, since the absorption spectrum of oxyhemoglobin $\left(\mathrm{HbO}_{2}\right)$ and deoxyhemoglobin $(\mathrm{Hb})$ are different, ${ }^{11}$ it is possible to retrieve information about changes of their concentration by monitoring the attenuation of constant intensity light source at multiple wavelengths after propagation in the tissue. Many of these cerebral oximeters perform multidistance measurements and the so-called spatially resolved spectroscopy algorithm, in order to quantify the absolute microvascular blood oxygen saturation $\left(\mathrm{StO}_{2}\right)$.

Cerebral oximetry using CW-NIRS has already been introduced as a clinical device in neonatology, and different randomized clinical trials have demonstrated that adding cerebral oximetry to the standard clinical care can reduce the burden of cerebral hypoxia in preterm infants. ${ }^{12,13}$ In spite of this, it is accepted in the neonatology community that further technical 
progress is still needed for a routine clinical use of oximeters. ${ }^{14}$ In particular, the current technology is deemed not precise enough, especially when repositioning the sensor over the infants head, with a variability over replacement measured $>5 \%$ in commercial oximeters. ${ }^{15}$ This, unfortunately, is too high for clinical purposes since it makes it difficult to discriminate between a safe value $(\sim 70 \%)$ and the threshold for intervention (usually 55\%). ${ }^{14} \mathrm{~A}$ related issue is that the absolute $\mathrm{StO}_{2}$ determination is device-dependent, which further complicates the use of thresholds to define a safe range. ${ }^{14}$

The European project BabyLux (January 2014 to April 2017) ${ }^{16}$ aims at introducing a solution for continuous neuromonitoring of infants at the cot-side by building a device based on more advanced forms of diffuse optical technologies. The goal is to achieve more precise, robust, and accurate results compared to the state-of-the-art methods. However, it also aims at adding a direct measurement of microvascular cerebral blood flow in order to estimate an index of cerebral oxygen metabolism. To this end, the BabyLux device has integrated time-resolved NIRS (TRS) and diffuse correlation spectroscopy (DCS). The former technology is an evolution of CW-NIRS. Instead of a constant intensity light source, it uses short laser pulses (about $100 \mathrm{ps}$ ) and studies their propagation in the tissue in several wavelengths. ${ }^{17}$ By modeling the shape of the collected curve, it is possible to obtain the tissue optical properties (absorption $\mu_{a}$ and reduced scattering coefficient $\mu_{s}^{\prime}$ ) as absolute values at each wavelength and, hence, absolute $\mathrm{Hb}$ and $\mathrm{HbO}_{2}$ concentrations. The efficient decoupling of these two effects allows the TRS estimation of $\mathrm{StO}_{2}$ to be more robust and accurate than what can be achieved with CW-NIRS. ${ }^{18}$

On the other hand, DCS measures the microvascular cerebral blood flow locally. ${ }^{19,20}$ It uses a long coherence length laser and measures the intensity fluctuations of a single, diffuse laser speckle resulting from the propagation of photons into the tissue. Those fluctuations are affected by the moving scatterers, dominated by red blood cells and can be used to obtain a blood flow index (BFI), which is proportional to the blood flow in the tissue. ${ }^{21}$

Therefore, a hybrid TRS and DCS device, like the BabyLux device, can provide simultaneous, independent, and absolute measurement of blood oxygenation and perfusion of the tissue, i.e., of both consumption by and supply of oxygen to the tissue. This allows for retrieving information on the metabolism, quantifying the relative measurement of cerebral metabolic rate of oxygen $\left(\mathrm{CMRO}_{2}\right){ }^{22}$ The possibility of employing such a hybrid device has been explored for more than a decade by employing different kinds of NIRS technologies for the blood oxygen saturation estimation. ${ }^{23-25}$ This approach has been particularly successful in newborns. ${ }^{26-30}$ However, most previous implementations were either specifically designed for newborns or "home-made" research devices, which are not particularly useful for extended, multicenter clinical testing. Recent emergence of other commercial devices is working toward changing this. ${ }^{31}$

Here, we describe the BabyLux device, which was built to be user-friendly and to be operated by clinical personnel without technical assistance. To demonstrate good performance in terms of precision and accuracy, we have performed tests in laboratory settings. Among the different characterization protocols that were suggested for the objective performance characterization of photon migration instruments, ${ }^{32-34}$ we have focused on the MEDPHOT protocols for TRS. The MEDPHOT protocol, ${ }^{32}$ which consists of measuring a set of homogeneous solid phantoms with different optical properties, was employed to assess the accuracy, precision, and stability of the optical property estimation. As far as DCS was concerned, we have employed measurements on a calibrated liquid phantom to define the performance in terms of precision and stability of dynamic properties (flow) estimation. Last, test measurements on an adult forearm during arterial cuff occlusion were performed to show results from in vivo measurements on a well-controlled and dynamic variable configuration.

\section{Methods}

\subsection{Hardware}

The BabyLux device can be used in two different configurations. Figure 1(a) shows the main unit, which contains the optical and electronic components and the screen in its desktop configuration. In addition, the main case can be supported by a custom cart, as depicted in Fig. 1(b). The cart consists of an upper drawer for storage purposes and a bottom drawer for an uninterruptible power source battery (HAT 1101S, Hectronica, Spain) and for a medical grade isolator transformer (REO España 2002 S.A.). Wheels make the device portable.

A schematic of the main optical and electronic components of the DCS and TRS module is presented in Fig. 1(c). The TRS module uses three pulsed laser diode heads at 685, 760, and $820 \mathrm{~nm}$ (PicoQuant GmbH, Germany, pulse duration $<100 \mathrm{ps}$ and average power $<1 \mathrm{~mW}$ ), driven by a compact pulse driver at $20 \mathrm{MHz}$ (PicoQuant GmbH, Germany). The electrical connection between the pulse driver and the laser heads has a different length for each of the three lasers, in order to introduce different temporal delays between the pulses. Each branch of the pulsed light is divided by a $96 \% / 4 \%$ fiber splitter (OZ Optics LTD., Canada). The $4 \%$ branch of the splitter is attenuated (OZ Optics LTD., Canada), combined into a single fiber connector (Lightech SRL, Italy) and sent directly to the detector (PicoQuant, Germany) [dashed red lines in Fig. 1(c)] to monitor the temporal drift of the TRS lasers. The $96 \%$ branch is directed to motorized software-controlled attenuators (OZ Optics LTD., Canada), and, then, to the front panel of the device. At the detection side, the light is collected by an optical coupler and delivered to the detector (PicoQuant, Germany). The optical coupler combines the signal and the reference branches and contains a triple bandpass filter (AHF analysentechnik AG, Germany) to reduce the detection of the ambient light and the cross talk by the DCS signal. The output of the photon-counting detector is fed to the time-correlated single-photon-counting board (PicoQuant GmbH, Germany) to reconstruct the distribution of time of flight (DTOF) of the detected photons.

DCS module employs a customized, external cavity, long coherence length continuous-wave laser diode operating at around $785 \mathrm{~nm}$ (HemoPhotonics, S.L., Spain). The laser light passes through a bandpass optical filter centered at $780 \mathrm{~nm}$ with a 20-nm bandwidth (Semrock Inc.). The filter's purpose is to cut the tails in the spectrum of DCS light, in order to achieve a more complete blocking by the bandpass filter in front of the TRS detector. A manual attenuator is adjusted in order to reach a power of $\sim 20 \mathrm{~mW}$ at the probe head. We note that different laser units from the same series have slightly different specifications and the procedures that were introduced in the BabyLux project could be used to characterize each unit in terms of adherence to the specifications. Two single photon avalanche detectors are 

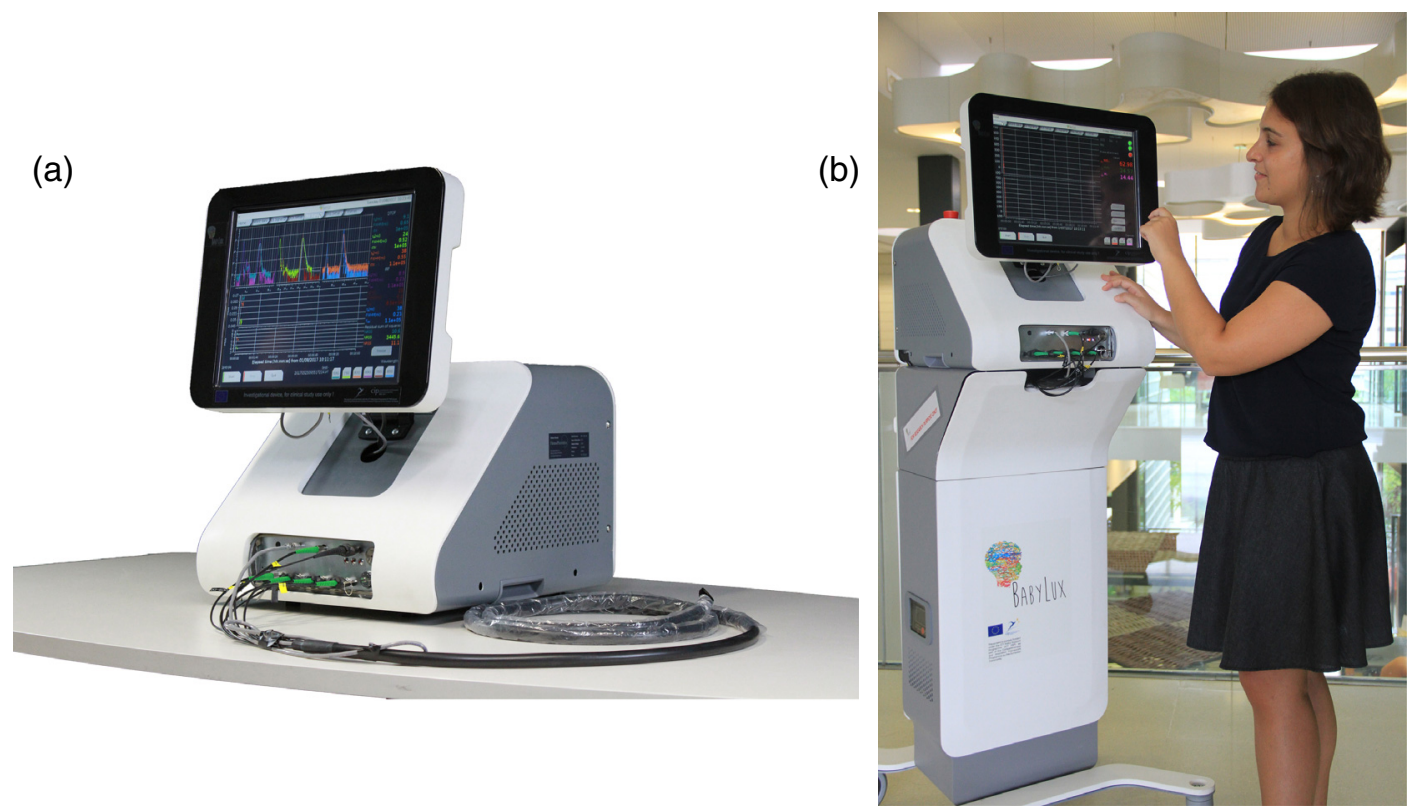

(c)

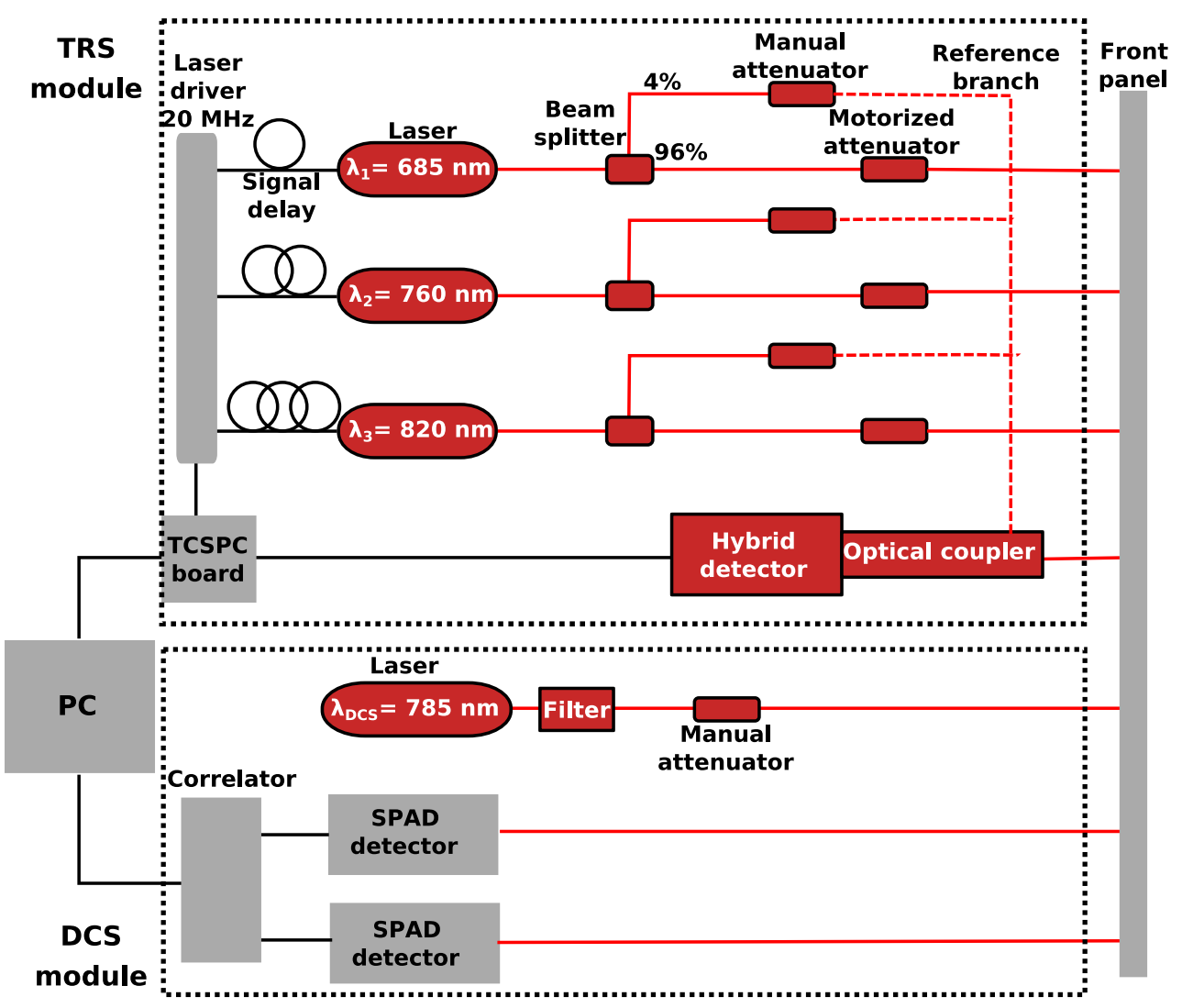

Fig. 1 (a) The BabyLux device on its desktop configuration, (b) a picture of the BabyLux device on the custom cart, and (c) schematic representation of the principal optical and electronic components of the BabyLux device.

used at the detector side (PicoQuant GmbH, Germany). Their input ferrule connector/physical contact (FC/PC) connectors are directly placed in the front panel of the device. The detectors' output is a train of transistor-transistor logic (TTL) pulses marking the arrival time of each photon. This is fed into a twochannel autocorrelation board (HemoPhotonics S.L., Spain) that calculates the intensity autocorrelation function of each signal.
The device is powered by two power supplies. An advanced technology extended medical grade power supply was used as the main one. This complies with the standard IEC 60601-1. Another $75 \mathrm{~W}(24 \mathrm{~V}, 3.2 \mathrm{~A})$ power supply was also added. This is not medical grade but the connection to mains is separated by a medical grade isolation transformer, likewise complying with IEC 60601-1. 


\subsection{Software}

A software developed by HemoPhotonics S.L. (Castelldefels, Spain) is used for the acquisition of both DCS and TRS measurements and for the communication with all the optical and electronic components of the device. The software, written in $\mathrm{C}++$ language and running in Linux (Kubuntu 14.04) environment, has been designed to be user-friendly. A normal user has access to the home window displayed in Fig. 2. The quality of optical data (e.g., the signal-to-noise ratio, stability, various parameters) is assessed online and is shown to the user with a color code (green for good, orange for intermediate, and red for low quality) in the top right corner. The online analysis of the data is performed by procedures provided by ICFO for DCS and Politecnico di Milano for TRS and the results of all the hemodynamic parameters and a plot following their time evolution are shown in real time. An example is given in Fig. 2 from the occlusion of an adult arm. Advanced users have access to other tabs, which show raw DCS and TRS signals. From these windows, it is also possible to change acquisition and analysis parameters.

All the measurements reported in this paper were acquired in $1 \mathrm{~s}$ averaging/accumulation time. TRS and DCS data were acquired simultaneously.

\subsection{Probe}

Four fibers are used for injecting light into the tissue. A single multimode fiber (400 $\mu \mathrm{m}$ core, $0.39 \mathrm{NA}$, Thorlabs, Germany) is employed for DCS and three graded index fibers $(62.5 \mu \mathrm{m}$ core,

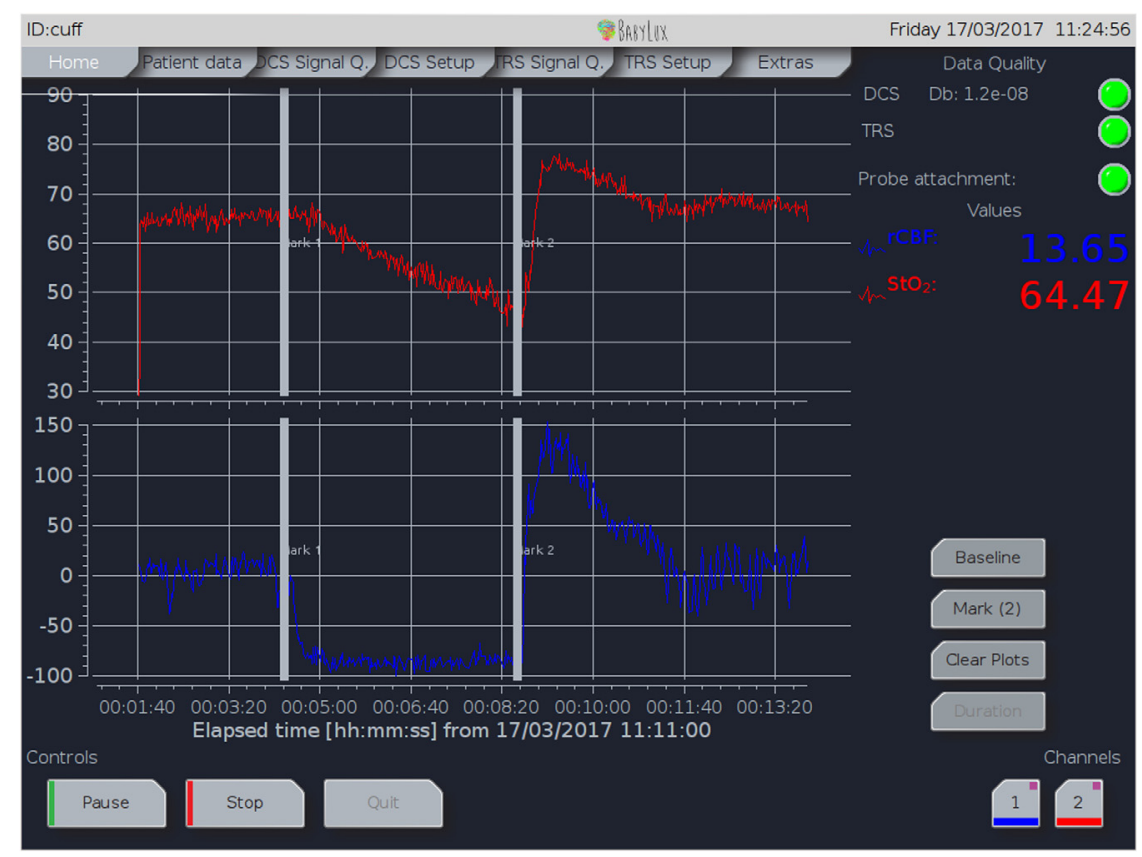

Fig. 2 Screenshot of the home window of the device during a cuff occlusion of an adult arm. Time series of the oxygen saturation (top) and relative BFI (bottom) are reported in the time plot. Color dots on the right top corner represent the optical quality of the data collected and the attachment of the probe as monitored by a capacitive sensor placed on the probe.

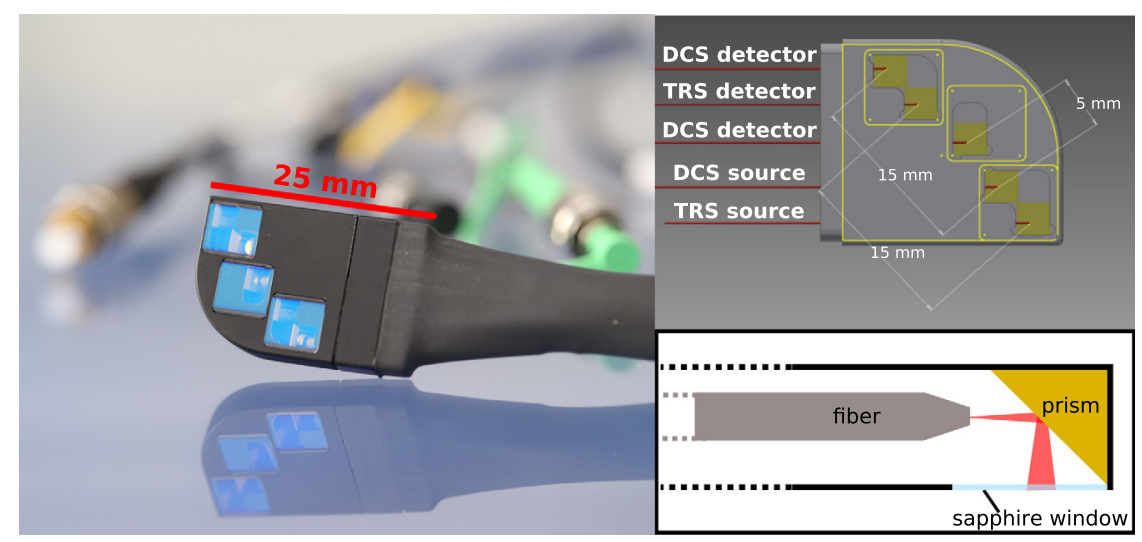

Fig. 3 Left side: a picture of the head of the probe is shown. Right side: a schematic representation highlighting the positions of the fibers and the distances between the pairs of source and detector fibers are shown. A schematic representation of the mounting of the fibers and the prisms is also presented. 
0.21 NA, Thorlabs, Germany) are employed for TRS, illuminating the same position. Two single-mode fibers $(4.4-\mu \mathrm{m}$ core, $0.13 \mathrm{NA}$, Thorlabs, Germany) are used as the detection fibers for DCS. A multimode graded index plastic optical fiber is used for TRS light detection (1-mm core, $0.3 \mathrm{NA}$, FIBERFINN).

A monolithic small and lightweight probe has been built by Fraunhofer Institute for Production Technology (IPT, Aachen, Germany) to encase all the seven fibers. A picture of the probe is presented in Fig. 3 with a schematic of the source and detector fiber positions. Prisms are used to deflect the light in the perpendicular direction with respect to the surface of the probe, which is sealed by sapphire windows to protect the fiber tips and prisms. For both DCS and TRS, the distance between source and detector is $15 \mathrm{~mm}$. A second DCS detector is placed at 5-mm distance from the DCS source to follow the changes in the superficial extracerebral tissue. We note that the layout of the source and detector fibers of the probe is flexible and other source-detector separations could be tested.

A capacitive sensor is implemented in the probe in order to check whether this is in contact with the skin. The state of the capacitive sensor is displayed in the home window, as shown in Fig. 2, with green and red to indicate attachment and displacement of the probe, respectively. This is also utilized for laser safety purposes.

The instrument response function (IRF) is measured by placing the probe over a reflective surface. A dedicated case to hold the probe in this position has been designed and built.

\subsection{Phantom Tests}

MEDPHOT protocol ${ }^{32}$ was applied to assess the performance of the TRS module using a phantom kit of twenty-four solid phantoms, with three different $\mu_{s}^{\prime}$ (nominal values 5, 10, $15 \mathrm{~cm}^{-1}$ at $660 \mathrm{~nm}$ ) and eight different $\mu_{a}$ (nominal values from 0 to $0.49 \mathrm{~cm}^{-1}$ in $0.07 \mathrm{~cm}^{-1}$ steps at $660 \mathrm{~nm}$ ).

This protocol is divided into five assays, a sixth one has been added since relevant for the clinical measurement planned with the BabyLux device. The six assays are as follows:

1. Accuracy: This test is designed to quantify the error made by the device on the optical property estimation. The error is defined as $\varepsilon=100 \times\left(x_{\text {meas }}-x_{\text {conv }}\right) /$ $x_{\text {conv }}$, where $x_{\text {meas }}$ is the quantity measured by the BabyLux device and $x_{\text {conv }}$ is the conventionally true value obtained by measuring each phantom with a broadband TRS system with high temporal resolution.

2. Linearity in $\mu_{a}$ and $\mu_{s}^{\prime}$ : Measured $\mu_{a}$ values are plotted against the conventionally true ones. In this case the measurements should ideally lie in a line passing through zero with a 45-deg slope. On the other hand, the measured $\mu_{s}^{\prime}$ are plotted against the conventionally true values of $\mu_{a}$. Therefore, they should ideally lie in three horizontal lines corresponding to the three conventionally true values of $\mu_{s}^{\prime}$.

3. Noise (or precision): Thirty continuous measurements are acquired on one phantom with nominal optical properties of $10 \mathrm{~cm}^{-1}$ for $\mu_{s}^{\prime}(690 \mathrm{~nm})$ and $0.1 \mathrm{~cm}^{-1}$ for $\mu_{a}(690 \mathrm{~nm})$ at different count levels, in the range from $10^{3}$ to $10^{8}$. To quantify the precision, we have used the coefficient of variation $(\mathrm{CV})$ over the 30 measurements $x=\left[x_{1}, \ldots, x_{30}\right]$, i.e., $\mathrm{CV}=100 \times \sigma(x) /\langle x\rangle$, where $\sigma(x)$ is the standard deviation and $\langle x\rangle$ is the averaged value.

4. Stability: The same phantom used in the previous point is measured for $\sim 10 \mathrm{~h}$ after the device has been switched on. The time series of the derived optical properties is plotted to check the time necessary for reaching their final values, i.e., the warming up of the device, and to check the presence of drifts or fluctuations.

5. Reproducibility: The same phantom is measured over five different days, at the same experimental conditions. Averaged value of optical properties over all the 5 days is calculated as well as the percentage deviation in each day with respect to them.

6. Reproducibility over probe replacement: The same phantom is measured over five different days, at the same experimental conditions. The probe is placed on the phantom, 30 measurements are acquired, then the probe is lifted, placed back on the phantom surface and another 30 acquisitions run. This is done until six sets of measurement are acquired. The CV over the six replacements is calculated for each of the 5 days.

All the described measurements are acquired collecting $10^{5}$ photon/s, if reachable, apart from point (3).

To test the DCS module, a water-based solution of polydisperse microparticles (HemoPhotonics S.L., Spain) ${ }^{35}$ has been employed. This phantom is used for the last three points of the above protocol (stability, reproducibility, and reproducibility over probe replacement). It has nominal optical properties of 0.17 and $7 \mathrm{~cm}^{-1}$ at $785 \mathrm{~nm}$ and a nominal particle diffusion coefficient of $1 \times 10^{-8} \mathrm{~cm}^{2} / \mathrm{s}$.

Finally, to quantify the possible mutual cross talk between TRS and DCS, we have acquired 30 TRS and DCS measurements on the same phantom both simultaneously (i.e., DCS on and TRS on) and independently (i.e., DCS on and TRS off, or vice versa). Optical and dynamic properties are estimated in the two modes and mean and standard deviation of the 30 measurements are calculated.

\subsection{In Vivo Protocol}

A protocol for arterial cuff occlusion of the arm was implemented on healthy adult volunteers to test the device in relevant settings closer to the operational ones. This test was previously proposed as a controlled and easy-to-repeat test to check the in vivo performance of cerebral oximeters. ${ }^{36}$

The studies were approved by the ethical committee of the Hospital Clinic Barcelona. Each subject signed an informed consent and the study was conducted according to the principles of the Declaration of Helsinki. The inclusion criteria was a skinfold thickness of $<8 \mathrm{~mm}$ as measured by a skin caliber. The probe was placed on the upper part of the flexor muscle of the lower arm. After 2 min of rest, arterial cuff occlusion at $230 \mathrm{mmHg}$ was maintained for $6 \mathrm{~min}$. The cuff was deflated and 7 more minutes were recorded. This protocol was repeated three times sequentially for each subject in the same session. 


\subsection{Data Analysis}

\subsubsection{Time-resolved near-infrared spectroscopy analysis}

To model the collected TRS data, the solution of the photon diffusion equation for the reflectance from a semi-infinite homogeneous medium is used. The following expression for the reflectance is obtained: ${ }^{37,38}$

$$
\begin{aligned}
R(t)= & \frac{v}{2 A}\left(\frac{1}{4 \pi D t}\right)^{3 / 2} \exp \left(-\frac{\rho^{2}}{4 D t}-\mu_{a} v t\right) \\
& \times\left[\exp \left(-\frac{z_{+}^{2}}{4 D t}\right)-\exp \left(\frac{z_{-}^{2}}{4 D t}\right)\right],
\end{aligned}
$$

where $v$ is the speed of light in the medium, $A$ accounts for the index mismatch between tissue and air. ${ }^{21,38,39}$ Here, $D$ is the photon diffusion coefficient $D=\frac{v}{3 \mu_{s}^{\prime}}, \rho$ is the distance between the injection point and the detection point, $z_{+}=z_{s}$ and $z_{-}=-2 z_{e}-z_{s}$, where $z_{s}=1 / \mu_{s}^{\prime}$ and $z_{e}=2 A / 3 \mu_{s}^{\prime}$. Here, $\mu_{a}$ and $\mu_{s}^{\prime}$ are the tissue optical properties, i.e., the absorption and the reduced scattering coefficients, respectively. The diffusion equation is a good model to describe the photon propagation into the tissue when $\mu_{a} \ll \mu_{s}^{\prime}$, which is usually valid for biological tissue.

The expression of the reflectance reported in Eq. (1) represents the response when the injected pulse is a delta function centered at time $t=0 \mathrm{ps}$, i.e., the Green's function solution. ${ }^{17,37,38}$

The reflectance of an experimental injected pulse $\tilde{R}(t)$ is given by the convolution of Eq. (1) with the IRF, i.e.,
$\tilde{R}(t)=R(t) \otimes I R F(t)$. Here, $\tilde{R}(t)$ is the model to be compared with the experimental DTOF, after normalizing by the curve area. A Levenberg-Marquardt procedure was employed to minimize the least square error between the two. The fitting range includes all the points with a number of counts higher than $90 \%$ of the peak value on the rising edge of the curve and $1 \%$ on the tail. The absorption and the reduced scattering coefficients are considered as the fitting parameters.

The time $t=0 \mathrm{ps}$ represents the photon launching time, and it is considered as the peak of the IRF in the realistic case when the injected pulse is not a delta pulse. This holds true if IRF is measured by directly facing the source and detector fibers. ${ }^{40}$ Since we have measured the IRF in a reflectance geometry, with some distance from the reflecting surface, a shift of the peak $t_{0}$ must be considered. This parameter is calibrated by measuring a solid phantom with known optical properties. We have used the above-explained model for the fitting procedure and a spectral fit was performed by defining a spectral constraint for the reduced scattering coefficient according to the Mie theory. Specifically, $\mu_{s}^{\prime}(\lambda)=a\left(\lambda / \lambda_{0}\right)^{-b},{ }^{41-43}$ where $\lambda_{0}$ is a reference wavelength and $a$ and $b$ are the parameters that depend on the scatterers size and density. Curves at the three wavelengths are then fitted simultaneously considering $\mu_{a}(\lambda)$, $a, b$, and $t_{0}$ as fitting parameters in order to define the best value for $t_{0}$.

Once $t_{0}$ was obtained for this system, the measured DTOF could be shifted with respect to the IRF and analyzed as explained above by fitting the DTOF with the model $\tilde{R}(t)$. An estimation of optical properties $\left[\mu_{a}(\lambda)\right.$ and $\left.\mu_{s}^{\prime}(\lambda)\right]$ for each wavelength was derived.
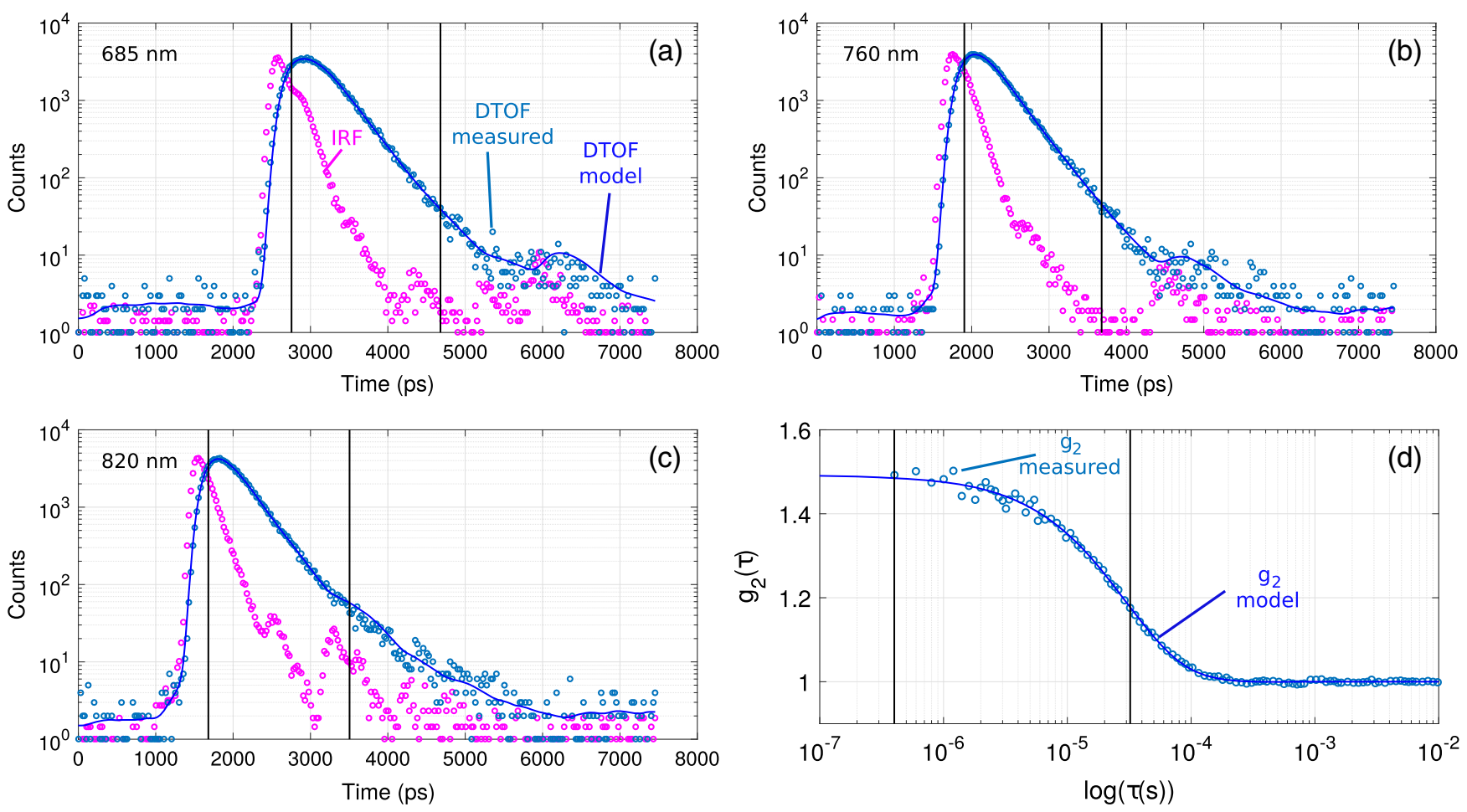

Fig. 4 IRF and an example of DTOF (collected from a solid phantom) are shown for the three wavelengths (a) $685 \mathrm{~nm}$, (b) $760 \mathrm{~nm}$, and (c) $820 \mathrm{~nm}$. The solid line represents the results of the fitting procedure. (d) A DCS-measured normalized, intensity autocorrelation curve collected from a liquid phantom is depicted, together with the results of the model, solid line. The black vertical lines highlight the region used for the fitting procedure. 


\subsubsection{Diffuse correlation spectroscopy analysis}

In the DCS module, the intensity fluctuations of the diffuse speckles are quantified by using a hardware correlator to calculate its normalized intensity autocorrelation function $\left[g_{2}(\tau), \tau\right.$ is the delay time]. The decay of this quantity depends on the movement of the moving scatterers, i.e., the red blood cells in case of most tissue measurements. ${ }^{19,21}$ The first step of the analysis process is to derive the normalized electric field autocorrelation function $g_{1}(\tau)$ from the measured $g_{2}(\tau)$, by the Siegert relation:

$g_{2}(\tau)=1+\beta\left|g_{1}(\tau)\right|^{2}$

where $\beta$ is a parameter that depends on the experimental conditions and can be estimated from the first points of the $g_{2}(\tau)$, i.e., $g_{2}(\tau=0)=1+\beta$. The experimentally derived $g_{1}(\tau)$ can be compared to the solution of the correlation diffusion equation by normalizing the electric field autocorrelation function $\left[G_{1}(\tau)\right]$, considering $g_{1}(\tau)=G_{1}(\tau) / G_{1}(0)$. As before, we have considered a semi-infinite homogeneous medium.
Therefore, the analytical expression of $G_{1}(\tau)$ is given as ${ }^{21}$

$G_{1}(\tau)=\frac{1}{4 \pi D}\left[\frac{e^{-K(\tau) r_{1}}}{r_{1}}-\frac{e^{-K(\tau) r_{e}}}{r_{e}}\right]$,

where the decay constant $K(\tau)=\sqrt{\left(\mu_{a}+2 \mu_{s}^{\prime} k_{0}^{2} B F I \tau\right) \frac{1}{D}}$, with $k_{0}$, the wavenumber of light into the medium, depending on the so-called BFI. This derivation assumes a Brownian model for the diffusion of the moving scatterers. ${ }^{20}$ BFI equals the particle diffuse coefficient $D_{B}$ multiplied by the fraction of moving scatterers to the total scatterers $(\alpha)\left(\mathrm{BFI}=\alpha D_{B}\right)$. In Eq. (3), $r_{1}=\sqrt{\rho^{2}+z_{+}^{2}}$ and $r_{e}=\sqrt{\rho^{2}+z_{-}^{2}}$ and $z_{+,-}$are defined in Sec. 2.6.1. BFI is extracted as fit parameter from Eq. (3), considering all the delay times for which $g_{2}(\tau)>0.6$. The optical properties measured by TRS are inserted as input parameters. Specifically, their average over the measurement period is used for phantom measurement. On the other hand, for the in vivo measurement, the whole time series of $\mu_{a}$ is inserted while $\mu_{s}^{\prime}$ is fixed to its baseline value.
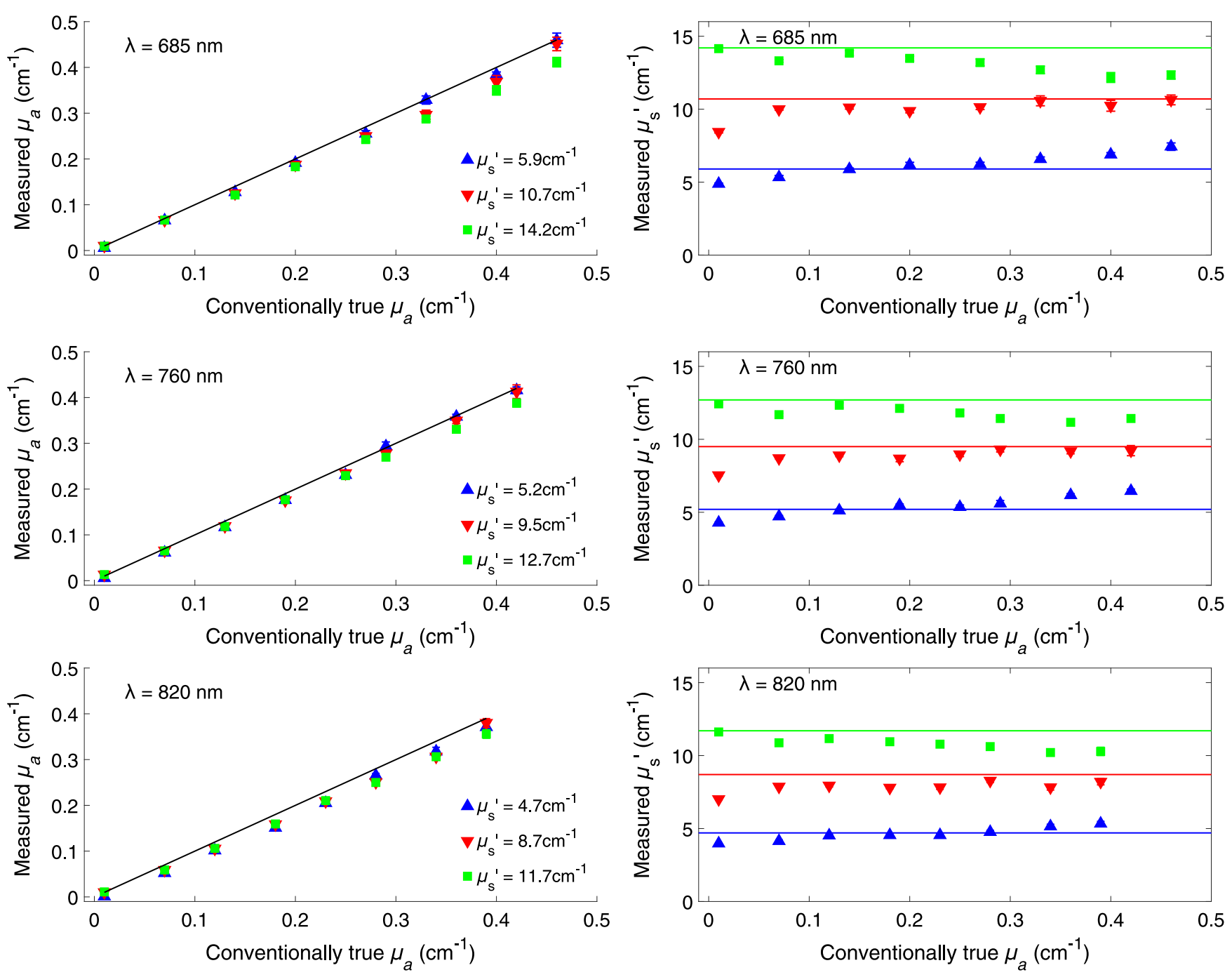

Fig. 5 Optical properties retrieved from a series of twenty-four solid phantoms, built with three different levels of reduced scattering coefficient and eight levels for absorption are shown. Each row represents one wavelength $(\lambda)$. In the left column, the measured $\mu_{a}$ are plotted against the conventionally true $\mu_{a}$; different colors and markers correspond to the different level of $\mu_{s}^{\prime}$. The black line represents the expected values. The right column reports the measured $\mu_{s}^{\prime}$ with respect to the conventionally true $\mu_{a}$. 


\subsubsection{In vivo measurement analysis}

From the retrieved values of absorption coefficients at 685,760 , and $820 \mathrm{~nm}$, the concentrations of $\mathrm{HbO}_{2}$ and $\mathrm{Hb}$ could be calculated. This is done assuming the linearity between absorption coefficients and the chromophore concentrations. In particular, $\mu_{a}(\lambda)=\sum_{i}^{N_{c}} \varepsilon_{i}(\lambda) c_{i}$, where $c_{i}$ is the concentration and $\varepsilon_{i}$ is the extinction coefficient of the $i$ 'th of the total $N_{c}$ chromophores. Since water content was fixed at the $70 \%$ of the total chromophore concentration, only two unknowns were present, and the system was overdetermined and could be solved by the method of the least squares.

As explained in the protocol section, three occlusions were performed for each subject. The response in each occlusion was characterized by extracting the level of $\mathrm{BFI}$ and $\mathrm{StO}_{2}$ reached during occlusion and after releasing the cuff. To test whether the response differed over multiple occlusions, a linear mixed effects (LME) model for those two variables was built with the number of occlusion as fixed effect and subject identifier as the random effect.

\section{Results}

Figure 4 shows examples of IRF and DTOF that were collected from a solid phantom with nominal optical properties of $10 \mathrm{~cm}^{-1}$ for $\mu_{s}^{\prime}$ and $0.1 \mathrm{~cm}^{-1}$ for $\mu_{a}$ at $690 \mathrm{~nm}$. The measured curves are shown for the three wavelengths used [(a) $685 \mathrm{~nm}$, (b) $760 \mathrm{~nm}$, and (c) $820 \mathrm{~nm}$ ], together with the results from the model. We have obtained optical properties of 0.079 and $11.6 \mathrm{~cm}^{-1}$ at $685 \mathrm{~nm}$, of 0.077 and $9.9 \mathrm{~cm}^{-1}$ at $760 \mathrm{~nm}$, and of 0.072 and $8.9 \mathrm{~cm}^{-1}$ at $820 \mathrm{~nm}$, for $\mu_{a}$ and $\mu_{s}^{\prime}$ coefficient, respectively. Figure 4(d) reports an example of a normalized intensity autocorrelation curve collected from a liquid phantom with $\mu_{a}(785 \mathrm{~nm})=0.17 \mathrm{~cm}^{-1}$ and $\mu_{s}^{\prime}(785 \mathrm{~nm})=7 \mathrm{~cm}^{-1}$, together with the modeled curve. The fitting procedure gives a BFI of $1.7 \times 10^{-8} \mathrm{~cm}^{2} / \mathrm{s}$.

\subsection{Phantom Tests}

The measurements were performed on the 24 solid phantoms within the framework of the MEDPHOT protocol and allowed
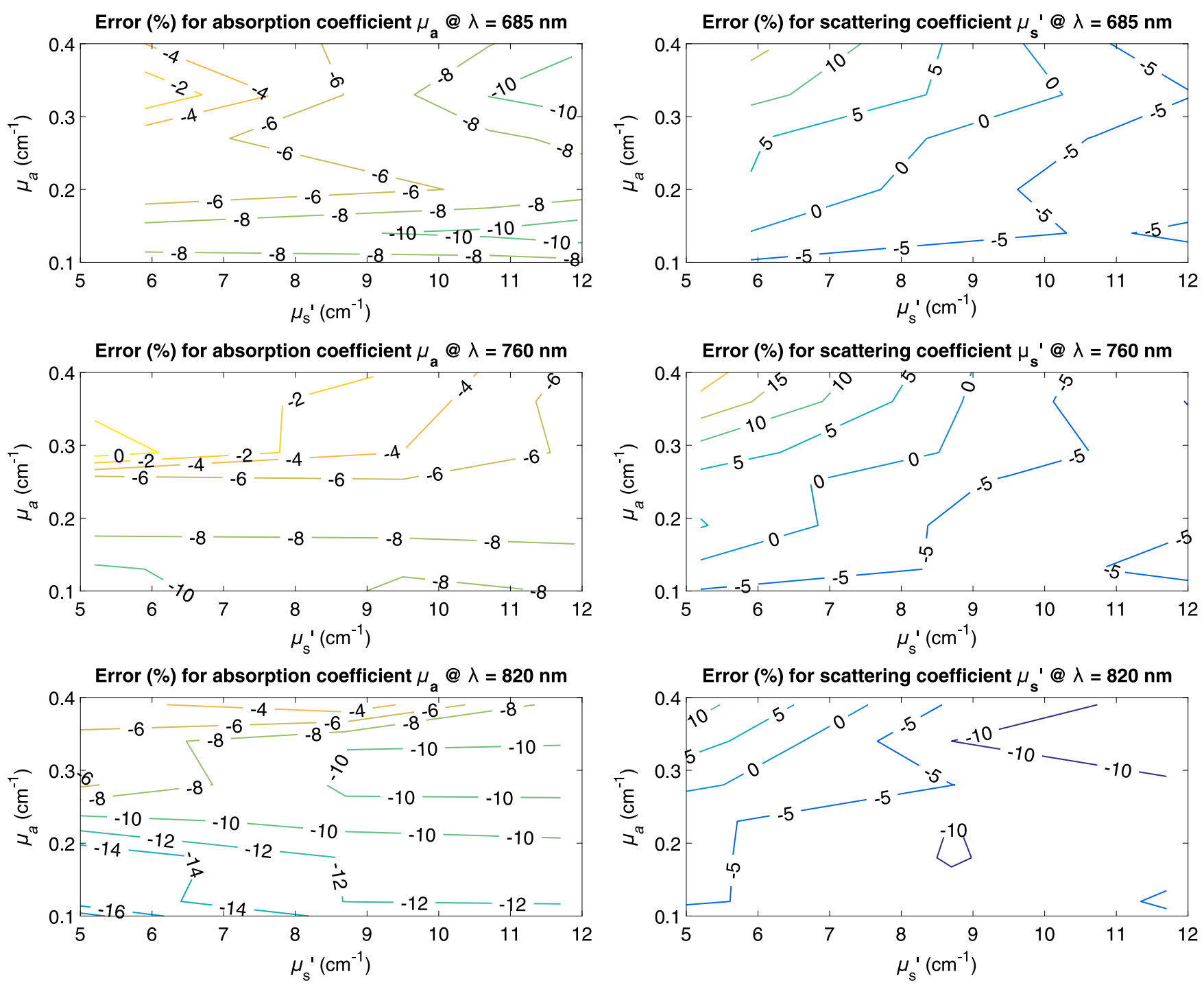

Fig. 6 Contour plots showing the errors in the estimated values with respect to the conventionally true values for $\mu_{a}$ (left column) and $\mu_{s}^{\prime}$ (right column) derived from the measurement of 24 solid phantoms. Each row represents one wavelength $(\lambda)$. 
the characterization of accuracy (assay 1) and linearity (assay 2) of the optical property estimation by TRS. Left column of Fig. 5 shows the linearity of the system for absorption measurement by showing the behavior of $\mu_{a}$ estimation with respect to the conventionally true values for $\mu_{a}$. The black line represents the expected values; therefore, the distance of each point from this line gives an indication of the accuracy of the estimation. These errors are quantified in the left column of Fig. 6 for $\mu_{a}$. If we focus on the values of interest of $\mu_{a}=0.2 \mathrm{~cm}^{-1}$ and $\mu_{s}^{\prime}=7 \mathrm{~cm}^{-1}$, the error is around $6 \%$ for 685 and $760 \mathrm{~nm}$, whereas it is $12 \%$ for $820 \mathrm{~nm}$. The values for errors lie in the expected range and are mainly due to the variation of the experimental conditions with respect to the diffusion model ${ }^{44}$

The right column of Fig. 5 reports the measured $\mu_{s}^{\prime}$ with respect to the conventionally true values for $\mu_{a}$ in order to show the coupling of $\mu_{s}^{\prime}$ to $\mu_{a}$. The horizontal lines represent the expected values for $\mu_{s}^{\prime}$ and the deviations from these lines are quantified in the right column of Fig. 6, showing the highest error for high value of $\mu_{a}$ and low values of $\mu_{s}^{\prime}$, as expected due to the deviation from the diffusion model. ${ }^{45}$ An error of about $10 \%$ for $\mu_{s}^{\prime}$ is obtained for the values of interest of $\mu_{a}=0.2 \mathrm{~cm}^{-1}$ and $\mu_{s}^{\prime}=7 \mathrm{~cm}^{-1}$; this is also comparable to what was expected due to the diffusion model. ${ }^{44}$

Results of assay 3 are reported in the top row of Fig. 7. The $\mathrm{CV}$ of optical property measurement at different count levels is reported in a logarithmic scale. A value of $1 \%$ is reached with $5 \times 10^{5}$ counts. Owing to the Poisson nature of the photon noise, the $\mathrm{CV}$ is expected to decrease as the square root of the counts. To test this, the logarithmic transformation of CV and the counts, as plotted in Fig. 7, was tested for linear regression. For each wavelength and for both $\mu_{a}$ and $\mu_{s}^{\prime}$, the $95 \%$ confidence interval of the slope contains -0.5 , i.e., a square root dependence, as expected.

On the other hand, the bottom row of Fig. 7 shows the results for assay 4. Optical properties estimated from $8 \mathrm{~h}$ of measurement right after the starting of the device are reported. The horizontal lines represent a range of $\pm 3 \%$ with respect to the average values calculated in the last hour of measurement. A warm-up period of $1 \mathrm{~h}$ is thus identified. After the warm-up period, no shifts or deviations were detected in the results.

By performing multiple measurements for different days, the reproducibility of the estimations over different days (assay 5) and over replacements of the probe on the phantom (assay 6) could be assessed. These results are reported in Table 1 . The deviation among different days of measurement is low, with a maximum value of $5 \%$ reached only in 1 day. $\mathrm{CV}$ over replacements is also low with a maximum of $2.5 \%$.

As for DCS, the results of measurement on a liquid phantom over $5 \mathrm{~h}$ are reported in Fig. 8. Retrieved BFI values show no trend over time and the CV over the whole period is $1.5 \%$. Subsequently, multiple measurements were done on a liquid phantom to access the repeatability over days and over replacements. Results are reported in Table 2. While results for CV over replacements are very good and similar to those obtained for TRS, the deviation from average value of BFI between days is larger than those for optical properties, reaching $8 \%$. It must be noted that variability of liquid phantom conditions plays an important role in dynamic property measurement, mainly because the measured diffusion coefficient depends on the environmental conditions, such as the temperature. ${ }^{46}$
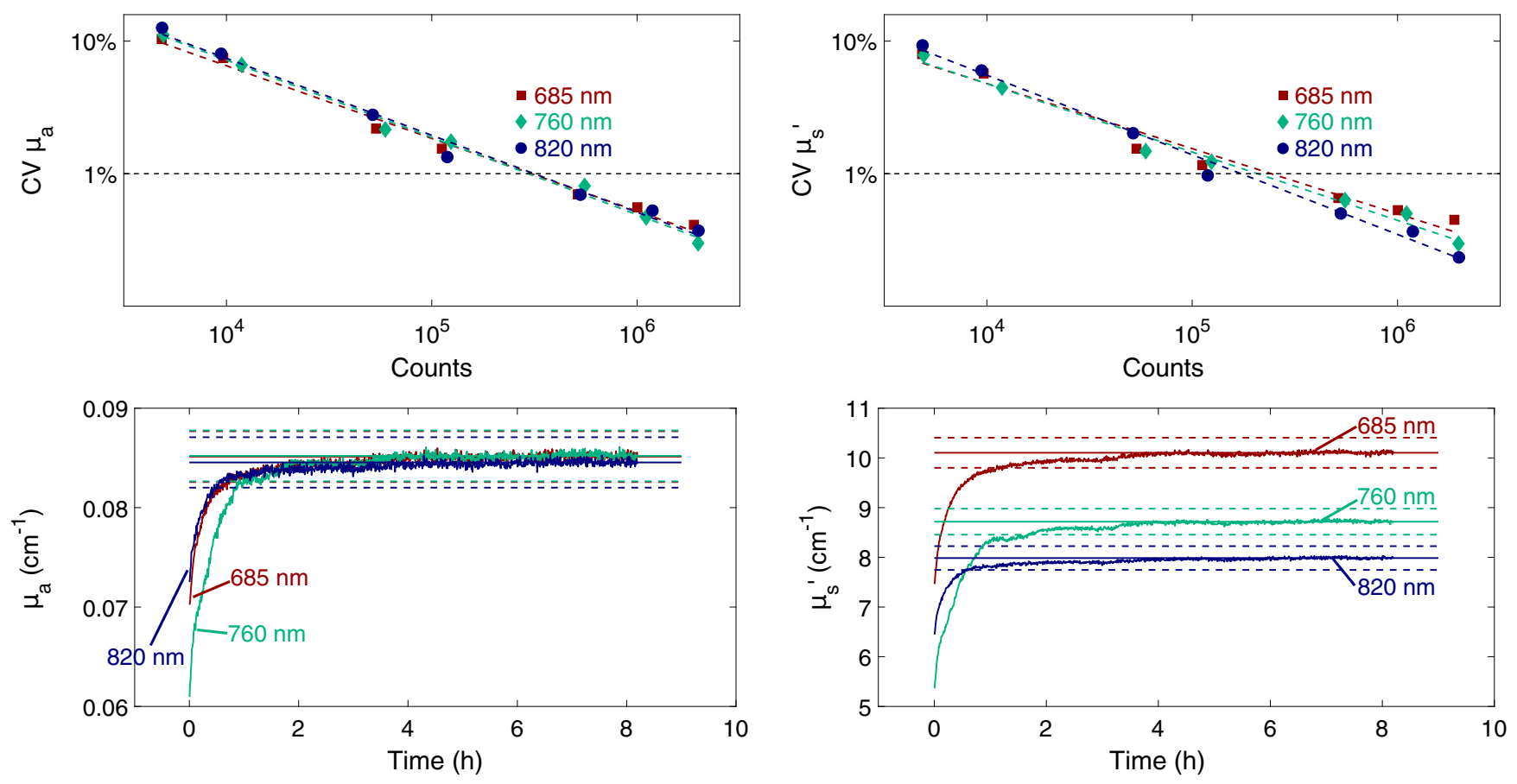

Fig. 7 Top row reports the $\mathrm{CV}$ of $\mu_{\mathrm{a}}$ and $\mu_{s}^{\prime}$ estimation over 30 continuous measurements at different count levels. Both axes are in logarithmic scale, dashed lines represent the linear regression, whose slope is -0.5 for all of them, as expected by Poisson photon noise. About $1 \%$ stability level is reached at $5 \times 10^{5}$ counts for the $\mu_{a}$ and already at $10^{5}$ counts for the $\mu_{s}^{\prime}$. The bottom row reports results of $\mu_{a}$ and $\mu_{s}^{\prime}$ over $8 \mathrm{~h}$ after turning on the device for the three wavelengths. The dashed horizontal lines represent the $\pm 3 \%$ range of the average value obtained from the last hour of measurement that is reached after $1 \mathrm{~h}$ of laser functioning. 
Table 1 The same solid phantom was measured on five different days, acquiring six measurements per day replacing each time the probe on the surface of the phantom. In the table, for each wavelength, the average value of optical properties is presented. In addition, the percentage deviation from the average values and the $\mathrm{CV}$ over replacements measured on each of the 5 days are reported.

\begin{tabular}{|c|c|c|c|c|c|c|c|c|}
\hline \multirow[b]{2}{*}{$\lambda(\mathrm{nm})$} & \multicolumn{4}{|c|}{$\mu_{a}$} & \multicolumn{4}{|c|}{$\mu_{s}^{\prime}$} \\
\hline & $\begin{array}{c}\text { Average } \\
\text { value }\left(\mathrm{cm}^{-1}\right)\end{array}$ & Day & $\begin{array}{c}\text { Deviation from } \\
\text { average (\%) }\end{array}$ & $\begin{array}{c}\text { CV over } \\
\text { replacement (\%) }\end{array}$ & $\begin{array}{l}\text { Average value } \\
\left(\mathrm{cm}^{-1}\right)\end{array}$ & Day & $\begin{array}{c}\text { Deviation from } \\
\text { average (\%) }\end{array}$ & $\begin{array}{c}\text { CV over } \\
\text { replacement (\%) }\end{array}$ \\
\hline \multirow[t]{5}{*}{685} & 0.078 & 1 & -0.5 & 2.3 & 11.5 & 1 & 1.2 & 1.8 \\
\hline & & 2 & -2.4 & 2.5 & & 2 & -0.6 & 1.9 \\
\hline & & 3 & 5.4 & 1.9 & & 3 & 0.9 & 1.5 \\
\hline & & 4 & 0.1 & 2.5 & & 4 & 0.2 & 1.9 \\
\hline & & 5 & -2.6 & 1.6 & & 5 & -1.7 & 1.3 \\
\hline \multirow[t]{5}{*}{760} & 0.076 & 1 & -0.04 & 2.2 & 9.7 & 1 & 1.1 & 1.6 \\
\hline & & 2 & -2.7 & 2.4 & & 2 & 0.2 & 1.5 \\
\hline & & 3 & 5.9 & 2.5 & & 3 & 1.0 & 2.0 \\
\hline & & 4 & -0.2 & 2.2 & & 4 & -0.2 & 1.5 \\
\hline & & 5 & -5.2 & 2.5 & & 5 & -3.8 & 1.7 \\
\hline \multirow[t]{5}{*}{820} & 0.073 & 1 & -0.6 & 2.3 & 8.7 & 1 & 0.8 & 1.3 \\
\hline & & 2 & -0.2 & 1.9 & & 2 & 2.8 & 1.4 \\
\hline & & 3 & 5.1 & 2.0 & & 3 & -0.5 & 1.4 \\
\hline & & 4 & 0.9 & 2.1 & & 4 & 0.7 & 1.6 \\
\hline & & 5 & -5.1 & 1.9 & & 5 & -3.8 & 1.3 \\
\hline
\end{tabular}

The final test performed on phantoms was aimed at assessing whether each module influences the other, to evaluate potential cross talk, since DCS and TRS measurements are acquired simultaneously. Table 3 compares the results of DCS measurements on the same liquid phantom both in the presence of TRS light shining from the probe and without the TRS light. Intensity $\beta$ parameter and BFI do not vary with and without TRS light shining from the probe, considering the standard deviation

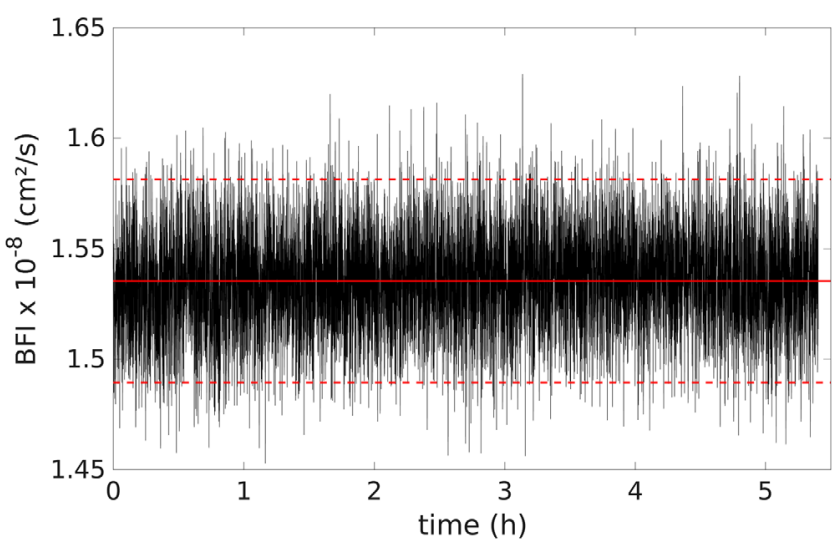

Fig. 8 BFI measurement of a liquid phantom by DCS over hours. The red continuous line indicates the average value, whereas the dashed line represents the $\pm 3 \%$ range. within the measurements. This is also confirmed for TRS. Table 4 shows the equivalent results for optical properties measured by TRS with and without DCS light shining from the probe. The results of the two modes overlap, considering the mean and the standard deviation of the 30 measurements. The increase of TRS background must be related to the counts in all the 2048 channels. It increases by only two counts per channels on average, which does not affect the measurement.

Table 2 The same liquid phantom was measured on five different days, acquiring six measurements per day replacing each time the probe on the surface of the phantom. The table reports the average value obtained for BFI and, for each day, the deviation from the average value and the CV over replacement.

\begin{tabular}{lccc}
\hline \multicolumn{3}{c}{ BFI } \\
\hline Average value & Day & $\begin{array}{c}\text { Deviation from } \\
\text { average }(\%)\end{array}$ & $\begin{array}{c}\text { CV over } \\
\text { replacement (\%) }\end{array}$ \\
\hline $1.76 \times 10^{-8} \mathrm{~cm}^{2} / \mathrm{s}$ & 1 & -8.3 & 1.5 \\
& 2 & -1.3 & 1.5 \\
& 3 & 7.0 & 1.0 \\
& 4 & 0.1 & 0.8 \\
& 5 & 2.5 & 1.0 \\
\hline
\end{tabular}


Table 3 The same liquid phantom is measured with and without TRS laser light shining from the probe, acquiring 30 curves. The table reports the mean $\mathrm{BFI}, \beta$, and intensity with the standard deviation (in brackets) over the 30 measurements.

\begin{tabular}{lccc} 
TRS light & $\mathrm{BFI}\left(\times 10^{-8} \mathrm{~cm}^{2} / \mathrm{s}\right)$ & $\beta$ & Intensity $(\mathrm{kHz})$ \\
\hline OFF & $1.95(0.09)$ & $0.50(0.01)$ & $153(2)$ \\
ON & $1.90(0.08)$ & $0.49(0.01)$ & $154(1)$ \\
\hline
\end{tabular}

Table 4 Thirty TRS curves are acquired from a solid phantom with nominal properties of $\mu_{a}=0.27 \mathrm{~cm}^{-1}$ and $\mu_{s}^{\prime}=10 \mathrm{~cm}^{-1}$ at $660 \mathrm{~nm}$ with and without DCS laser light shining from the probe. The table reports the mean optical properties with the standard deviation (in brackets) over the 30 measurements, as well as the total background noise, registered in all the 2048 channels.

\begin{tabular}{lcccc}
\hline \multicolumn{1}{c}{ DCS } & & & \\
\hline$(\mathrm{nm})$ & light & $\mu_{a}\left(\mathrm{~cm}^{-1}\right)$ & $\mu_{s}^{\prime}\left(\mathrm{cm}^{-1}\right)$ & $\begin{array}{c}\text { Total background } \\
\text { counts }\end{array}$ \\
\hline 685 & OFF & $0.243(0.002)$ & $9.49(0.06)$ & 6144 \\
& ON & $0.242(0.002)$ & $9.42(0.09)$ & 8192 \\
760 & OFF & $0.232(0.002)$ & $8.38(0.06)$ & 4096 \\
& ON & $0.232(0.003)$ & $8.35(0.09)$ & 8192 \\
820 & OFF & $0.215(0.002)$ & $7.55(0.05)$ & 4096 \\
& ON & $0.214(0.002)$ & $7.49(0.07)$ & 8192 \\
\hline
\end{tabular}
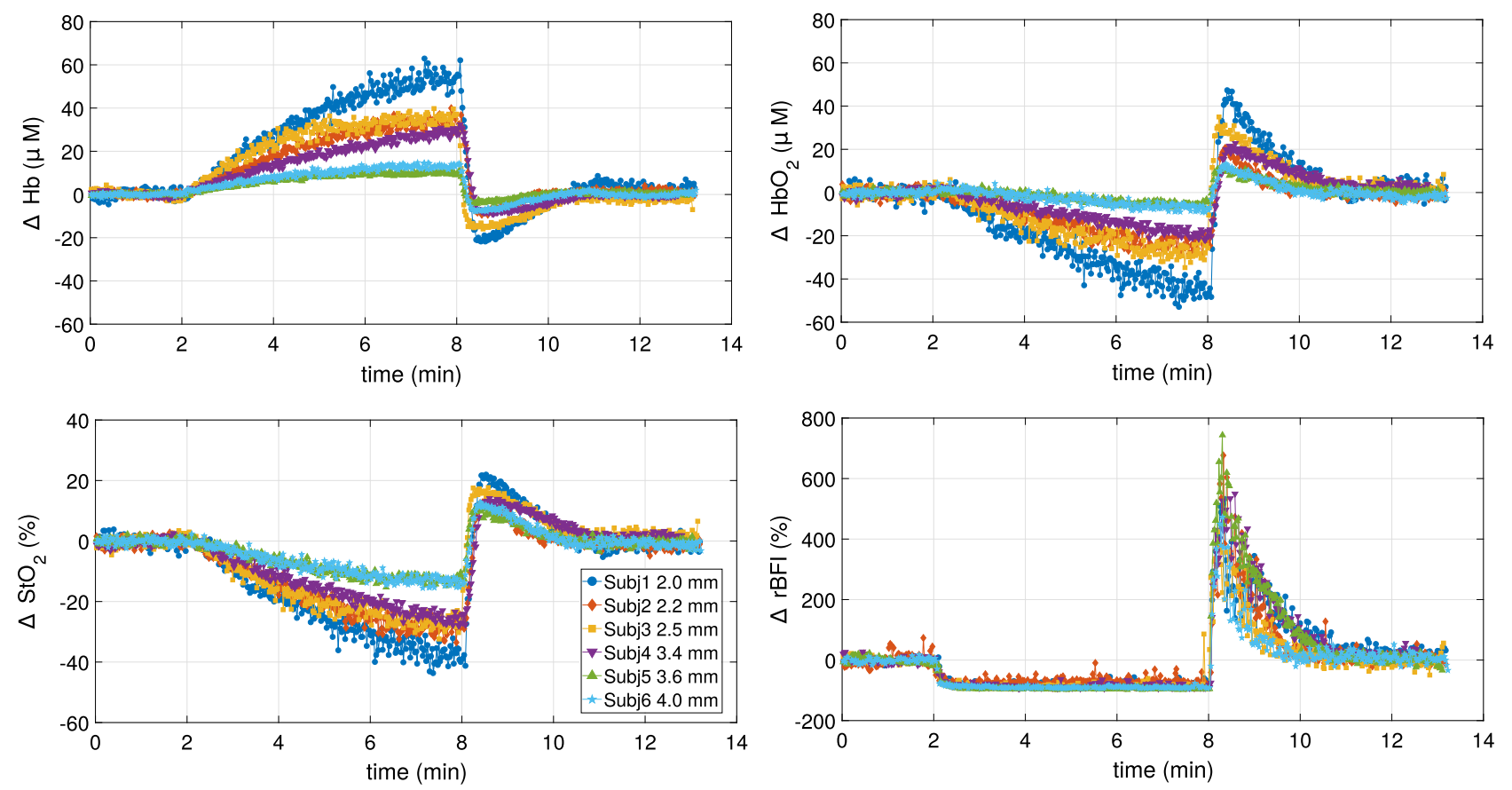

Fig. 9 Time series of changes of $\mathrm{Hb}, \mathrm{HbO}_{2}, \mathrm{StO}_{2}$, and $\mathrm{rBFI}$ during the cuff occlusion protocol. Curves are reported for each subject, as color coded. Legend identifies the thickness for the superficial layer for each subject. 


\section{Discussion and Conclusion}

The BabyLux device is an optical neuromonitor built and developed primarily for employment in neonatology. It integrates DCS and TRS technologies to acquire independent and simultaneous measurements of cerebral perfusion and blood oxygenation. The BabyLux project, in the framework within which the device was developed, was motivated by the current clinical need for a technology that can continuously monitor the cerebral well-being of the neonate brain at the cot-side. Our goal was to evaluate the device performance in a quantitative manner, independent of the measurements on the infant brain. This work demonstrates that the BabyLux device is a good candidate for monitoring cerebral hemodynamics in the infant brain for two reasons. First, by using DCS, it has a direct measurement of the blood flow to the cerebral tissue. Instead of having information only on the consumption of oxygen, as done by NIRS that measures blood oxygen saturation, the BabyLux device provides a direct independent measurement of the supply of oxygen. Second, TRS allows for absolute measurement of optical properties and, consequently, of absolute $\mathrm{Hb}$ and $\mathrm{HbO}_{2}$ concentrations and blood oxygen saturation.

To improve the state-of-the-art method, the BabyLux device had to be not only compact, portable, and easy to use for the clinical personnel but also robust, precise, and accurate. For this reason, tests in the laboratory settings were used to assure good performance in the near-ideal scenario.

The results reported in this paper were obtained by an off-line analysis. Nonetheless, we note that the online analysis implemented in the device follows the same routine and gives the same results, provided that the same values are chosen for the input analysis parameters. The range of those values is to be defined during the clinical testing of the device, which is the scope of other works.

It is fundamental to assess the accuracy in estimation of the optical properties for different reasons. First, the absolute measurement of $\mathrm{Hb}$ and $\mathrm{HbO}_{2}$ concentrations and consequently of $\mathrm{StO}_{2}$ depends on the possibility to measure the absolute values of the optical properties of the tissue, in other words, on decoupling $\mu_{a}$ and $\mu_{s}^{\prime}$. In addition, the optical property values are needed for the BFI estimation since they are the input parameters of DCS analysis. Results over different solid phantoms show good performance in terms of accuracy, with an error of around $10 \%$ in the region of interest, comparable with other TRS devices performance ${ }^{32}$ and with the intrinsic limits of employing the photon diffuse equation. ${ }^{44,45}$

When acquiring the TRS measurements at different count levels, a variability of $1 \%$ is reached with $5 \times 10^{5}$ counts. This is in line with previous experimental tests on TRS systems ${ }^{32,44}$ that reported that the CV for the fitted optical properties decreases with the count level with a square root power law. This is expected by Poisson statistics for photon counts and is confirmed by our results. Accordingly, the variability can be lowered significantly by increasing the count level (e.g., $\sim 0.3 \%$ with $10^{6}$ counts). However, the limit of $10^{5}$ counts can be easily reached by the TRS system with short $(<1 \mathrm{~s})$ acquisition time, whereas $10^{6}$ could require a longer acquisition time or averaging of several DTOFs at the post-processing level. Therefore, we have decided to target $10^{5}$ counts in a 1 -s acquisition.

A standard procedure to assess the accuracy of the DCS measurement does not exist, mainly due to the complexity of making a stable liquid phantom. A mixture of water, ink, and lipid emulsions is usually used. Few techniques have been considered to change the particle diffusion coefficient in a controlled way, from changing the viscosity of the medium, its temperature, or adding forced motion by pumps. ${ }^{20,47-50}$ However, the phantoms for dynamic properties are still to be improved.

Therefore, only stability and reproducibility was tested for both modalities. Optical and dynamic properties of the phantoms were stable (within $3 \%$ ) over more than $5 \mathrm{~h}$ of measurements; drifts and significant fluctuations were not observed. The warm-up period of about $90 \mathrm{~min}$ was defined for TRS lasers. Measurements over different days of operation on the same phantom showed repeatable results, with a deviation of $<5 \%$. The variability over the replacement of the probe on the phantoms was also assessed, motivated by the fact that poor reproducibility in this scenario is a major drawback of current NIRS technologies. The performance in the ideal scenario of the laboratory settings showed good reproducibility over replacement both for TRS and DCS measurements with a CV over replacement $<2.5 \%$. It is now necessary to assess the performance of the device in a realistic scenario. Toward that end, the variability over replacement of the probe has been measured on infants in clinical settings. These results will be published independently. The present work sets the realistic limits to be expected from the more complex clinical studies.

An arterial cuff occlusion protocol was designed in order to test the device during in vivo measurement, in particular, to illustrate its performance and sensitivity when blood flow and saturation levels vary over a dynamic and wide range. The hemodynamic parameters changed in the expected manner. Within all the subjects, a wide range of level for $\mathrm{StO}_{2}$ was reached during the occlusion period, reflecting, presumably, differences in the oxygen metabolism of the muscles of those subjects. Furthermore, the decrease of $\mathrm{StO}_{2}$ was dependent on the measured thickness of the superficial layer, which is not surprising since the source-detector separation is optimized for probing relatively shallow regions, such as the infant brain. During the occlusion, the reduction of $\mathrm{StO}_{2}$ was larger in subjects with a thinner superficial layer since the active muscle tissue occupied a larger percentage of the probed volume with respect to subjects with a thicker superficial layer. The $\mathrm{StO}_{2}$ decrease reached $40 \%$ in the subject with a skin and adipose tissue layer of $2 \mathrm{~mm}$. It has been proven that for the thin superficial layer (7 $\mathrm{mm}$ for a source-detector separation of $20 \mathrm{~mm}$ ), the absorption properties retrieved using the model for homogeneous level are independent on the changes of the upper layer. ${ }^{51}$ Even if this holds true, $\mathrm{StO}_{2}$ changes depend on the amount of muscle tissue probed by the optics, which consumes more oxygen with respect to the skin and adipose tissues, as it was demonstrated in a previous work changing the sourcedetector separation on the same subjects. ${ }^{52}$

The blood flow increase after the release of the cuff was homogeneous within the subjects and did not depend on the superficial layer thickness. Specifically, it increased by $490 \%$ as the median over the sample population with a narrow interquartile range of $380 \%$ and $510 \%$. In the previously cited work, the blood flow level of hyperemia was actually dependent on the source-detector separation. ${ }^{52}$ It must be noted that the occlusion in the mentioned work was kept for less time ( 3 min instead of $6 \mathrm{~min}$ ) and the blood flow increase was far lower than in our protocol (threefold increase instead of fivefold). Different lengths of occlusion may be the source of the discrepancy and the more extreme hypoxia due to the longer occlusion may be the cause of the fact that the response was not dependent 
on the superficial layer thickness. Since it has been proven that the depth sensitivity of DCS was higher than CW-NIRS and the contrast-to-noise ratio of the deeper layer change was equivalent, ${ }^{53}$ we did not expect DCS to be less sensitive to the muscle hemodynamics than TRS in this measurement.

TRS is not the only diffuse optical technology that can measure absolute optical properties. It has been shown that this can be done by a broadband CW-NIRS system. ${ }^{54}$ However, this relies on a more complex calibration scheme and as all continuous-wave methods are more prone to influences of external light. Consequently, this is often used to measure baseline optical properties and it relies on Beer-Lambert law for measuring change from baseline. Traditionally, the other technology that measures absolute optical properties are frequency-domain NIRS (FD-NIRS), which uses intensity-modulated light to probe the tissue. A commercial system integrating FD-NIRS and DCS is in the market and has been previously reported. ${ }^{31}$ A few aspects of TRS technology makes it preferable over traditional FD-NIRS. The latter needs multidistance measurements in its most common implementation or the use of more complex multiple source modulation frequency measurements in order to retrieve the absolute values of the optical properties. In the former case, FD-NIRS may be affected by the heterogeneity of the probed tissue and the probes are also more complex and larger. In the latter case, the complexity of the multifrequency approach has prevented the commercialization of such FD-NIRS systems.

On the other hand, TRS works with a single source-detector pair and from a single measurement allows for the estimation of the optical properties. In addition, the penetration depth of the TRS measurement does not depend on the source-detector separation and the depth sensitivity is naturally encoded in time. ${ }^{55-57}$ It has been demonstrated both theoretically ${ }^{58}$ and experimentally ${ }^{59}$ that TRS has a better penetration depth than FD-NIRS. It is true that TRS technology is traditionally more expensive but recent research has been focused on advancing the technology in order to build simpler and cheaper devices. ${ }^{60}$ Time will tell the ultimate preferred technology for a given application.

In conclusion, the laboratory tests showed good performance of the BabyLux device. The estimation of optical properties was accurate and precise. The results were stable over several hours of functioning, and they were repeatable within days and replacement of the probe. Good performance in the dynamic range and in vivo measurement has been proved by an arterial cuff occlusion protocol of the adult forearm. The performance in clinical settings, on infants, is currently being assessed with ongoing clinical studies.

\section{Disclosures}

Turgut Durduran is an inventor on a relevant patent (Patent US8082015B2, "Optical measurement of tissue blood flow, hemodynamics and oxygenation"). ICFO has equity ownership in the spin-off company HemoPhotonics S.L. Potential financial conflicts of interest and objectivity of research have been monitored by ICFO's Knowledge \& Technology Transfer Department. No financial conflicts of interest have been identified. Udo Weigel is the CEO, has equity ownership in HemoPhotonics S.L., and is an employee in the company. His role in the project has been defined by the project objectives, tasks, and work packages and has been reviewed by the European Commission.

\section{Acknowledgments}

This research was funded by the European Commission Competitiveness for Innovation Program (Grant Agreement No. 620996) as part of the project "An optical neuromonitor of cerebral oxygen metabolism and blood flow for neonatology (BabyLux)". We also acknowledge funding from Fundació CELLEX Barcelona, Ministerio de Economía y Competitividad/FEDER (PHOTODEMENTIA, DPI2015-64358-C2-1-R), Instituto de Salud CarlosIII/FEDER (MEDPHOTAGE, DTS16/ 00087), the "Severo Ochoa" Programme for Centres of Excellence in R\&D (SEV-2015-0522), the Obra social "la Caixa" Foundation (LlumMedBcn), Institució CERCA, AGAUR-Generalitat (2017 SGR 1380), and LASERLABEUROPE IV (GA: 654148). Martina Giovannella's PhD was funded by the "Severo Ochoa" Programme for Centres of Excellence in R\&D (SEV-2015-0522).

\section{References}

1. March of Dimes, PMNCH, Save the Children, and WHO, Born Too Soon - The Global Action Report on Preterm Birth, World Health Organization, Geneva (2012).

2. S. Saigal and L. W. Doyle, "An overview of mortality and sequelae of preterm birth from infancy to adulthood," Lancet 371, 261-269 (2008).

3. S. Noori, T. A. Stavroudis, and I. Seri, "Systemic and cerebral hemodynamics during the transitional period after premature birth," Clin. Perinatol. 36(4), 723-736 (2009).

4. N. Brew, D. Walker, and F. Y. Wong, "Cerebral vascular regulation and brain injury in preterm infants," Am. J. Physiol. Regul. Integr. Comp. Physiol. 306, R773-R786 (2014).

5. T. Austin, "Measurement of cerebral oxygenation in preterm infants: is it useful?" Dev. Med. Child Neurol. 57(5), 404-405 (2015).

6. G. Greisen, T. Leung, and M. Wolf, "Has the time come to use nearinfrared spectroscopy as a routine clinical tool in preterm infants undergoing intensive care?" Philos. Trans. A. Math. Phys. Eng. Sci. 369, 4440-4451 (2011).

7. A. A. Garvey and E. M. Dempsey, "Applications of near infrared spectroscopy in the neonate," Curr. Opin. Pediatr. 30(2), 209-215 (2018).

8. M. Wolf, M. Ferrari, and V. Quaresima, "Progress of near-infrared spectroscopy and topography for brain and muscle clinical applications," J. Biomed. Opt. 12(6), 062104 (2007).

9. F. Scholkmann et al., "A review on continuous wave functional nearinfrared spectroscopy and imaging instrumentation and methodology," Neuroimage 85, 6-27 (2014).

10. A. Yodh and B. Chance, "Spectroscopy and imaging with diffusing light," Phys. Today 48(3), 34-40 (1995).

11. J. Kim, M. Xia, and H. Liu, "Extinction coefficients of hemoglobin for near-infrared spectroscopy of tissue," IEEE Eng. Med. Biol. Mag. 24, 118-121 (2005).

12. G. Pichler et al., "Cerebral oxygen saturation to guide oxygen delivery in preterm neonates for the immediate transition after birth: a 2-center randomized controlled pilot feasibility trial," J. Pediatr. 170, 73-78e4 (2016).

13. S. Hyttel-Sorensen et al., "Cerebral near infrared spectroscopy oximetry in extremely preterm infants: phase II randomised clinical trial," $B M J$ 350, g7635-g7635 (2015).

14. G. Greisen et al., "Cerebral oximetry in preterm infants: an agenda for research with a clear clinical goal," Neurophotonics 3(3), 031407 (2016).

15. L. C. Sorensen and G. Greisen, "Precision of measurement of cerebral tissue oxygenation index using near-infrared spectroscopy in preterm neonates," J. Biomed. Opt. 11(5), 054005 (2006).

16. BabyLux, "An optical neuro-monitor of cerebral oxygen metabolism and blood flow for neonatology," http://www.babylux-project.eu/ (2019).

17. M. Patterson, B. Chance, and B. Wilson, "Time resolved reflectance and transmittance for the noninvasive measurement of tissue optical properties," Appl. Opt. 28(12), 2331-2336 (1989).

18. A. Torricelli et al., "Time domain functional NIRS imaging for human brain mapping," Neuroimage 85, 28-50 (2014). 
19. D. A. Boas, L. E. Campbell, and A. G. Yodh, "Scattering and imaging with diffuse temporal field correlation," Phys. Rev. Lett. 75(9), 18551858 (1995).

20. D. A. Boas and A. G. Yodh, "Spatially varying dynamical properties of turbid media probed with diffusing temporal light correlation," J. Opt. Soc. Am. A 14, 192-215 (1997).

21. T. Durduran et al., "Diffuse optics for tissue monitoring and tomography," Reports Prog. Phys. 73(7), 076701 (2010).

22. T. Durduran and A. G. Yodh, "Diffuse correlation spectroscopy for non-invasive, micro-vascular cerebral blood flow measurement," Neuroimage 85, 51-63 (2014).

23. D. A. Boas, "Diffuse photon probes of structural and dynamical properties of turbid media: theory and biomedical applications," $\mathrm{PhD}$ thesis, University of Pennsylvania (1996).

24. C. Cheung et al., "In vivo cerebrovascular measurement combining diffuse near-infrared absorption and correlation spectroscopies," Phys. Med. Biol. 46(8), 2053-2065 (2001).

25. T. Durduran, "Non-invasive measurements of tissue hemodynamics with hybrid diffuse optical methods," PhD thesis, University of Pennsylvania (2004).

26. E. M. Buckley et al., "Cerebral hemodynamics in preterm infants during positional intervention measured with diffuse correlation spectroscopy and transcranial Doppler ultrasound," Opt. Express 17(15), 1257112581 (2009).

27. T. Durduran et al., "Optical measurement of cerebral hemodynamics and oxygen metabolism in neonates with congenital heart defects," J. Biomed. Opt. 15(3), 037004 (2010).

28. V. Jain et al., "Cerebral oxygen metabolism in neonates with congenital heart disease quantified by MRI and optics," J. Cereb. Blood Flow Metab. 34(3), 380-388 (2014).

29. P. Y. Lin et al., "Reduced cerebral blood flow and oxygen metabolism in extremely preterm neonates with low-grade germinal matrix- intraventricular hemorrhage," Sci. Rep. 6, 25903 (2016).

30. P. Farzam et al., "Shedding light on the neonatal brain: probing cerebral hemodynamics by diffuse optical spectroscopic methods," Sci. Rep. 7, 15786 (2017).

31. S. A. Carp et al., "Combined multi-distance frequency domain and diffuse correlation spectroscopy system with simultaneous data acquisition and real-time analysis," Biomed. Opt. Express 8(9), 3993-4006 (2017).

32. A. Pifferi et al., "Performance assessment of photon migration instruments: the MEDPHOT protocol," Appl. Opt. 44(11), 2104-2114 (2005).

33. H. Wabnitz et al., "Performance assessment of time-domain optical brain imagers, part 1: basic instrumental performance protocol," J. Biomed. Opt. 19(8), 086010 (2014).

34. H. Wabnitz et al., "Performance assessment of time-domain optical brain imagers, part 2: nEUROPt protocol," J. Biomed. Opt. 19(8), 086012 (2014).

35. M. Pagliazzi et al., "Long-lasting, liquid phantom for diffuse optical and correlation spectroscopies," in Biomed. Opt. 2016, Washington, D.C., OSA, p. JTu3A.25 (2016).

36. S. Hyttel-Sorensen et al., "Tissue oximetry: a comparison of mean values of regional tissue saturation, reproducibility and dynamic range of four NIRS-instruments on the human forearm," Biomed. Opt. Express 2(11), 3047-3057 (2011).

37. D. Contini, F. Martelli, and G. Zaccanti, "Photon migration through a turbid slab described by a model based on diffusion approximation. I. Theory," Appl. Opt. 36(19), 4587-4599 (1997).

38. F. Martelli et al., Light Propagation through Biological Tissue, SPIE Press, Bellingham, Washington (2010).

39. R. C. Haskell et al., "Boundary conditions for the diffusion equation in radiative transfer," J. Opt. Soc. Am. A 11, 2727-2741 (1994).

40. V. Ntziachristos and B. Chance, "Accuracy limits in the determination of absolute optical properties using time-resolved NIR spectroscopy," Med. Phys. 28(6), 1115-1124 (2001).

41. J. R. Mourant et al., "Predictions and measurements of scattering and absorption over broad wavelength ranges in tissue phantoms," Appl. Opt. 36(4), 949-957 (1997).

42. A. M. Nilsson et al., "Changes in spectral shape of tissue optical properties in conjunction with laser-induced thermotherapy," Appl. Opt. 37(7), 1256-1267 (1998).
43. S. L. Jacques and B. W. Pogue, "Tutorial on diffuse light transport," J. Biomed. Opt. 13(4), 041302 (2008).

44. R. Cubeddu et al., "Experimental test of theoretical models for timeresolved reflectance," Med. Phys. 23, 1625-1633 (1996).

45. L. Spinelli et al., "Accuracy of the nonlinear fitting procedure for timeresolved measurements on diffusive phantoms at NIR wavelengths," Proc. SPIE 7174, 717424 (2009).

46. A. Einstein, Investigations on the Theory of the Brownian Movement, Dover Publications, New York (1956).

47. L. Gagnon et al., "Investigation of diffuse correlation spectroscopy in multi-layered media including the human head," Opt. Express 16(20), 15514-15530 (2008).

48. J. G. Kim and H. Liu, "Investigation of biphasic tumor oxygen dynamics induced by hyperoxic gas intervention: the dynamic phantom approach," Appl. Opt. 47(2), 242-252 (2008).

49. Y. Lin et al., "Noncontact diffuse correlation spectroscopy for noninvasive deep tissue blood flow measurement," J. Biomed. Opt. 17(1), 010502 (2012).

50. L. Cortese et al., "Liquid phantoms for near-infrared and diffuse correlation spectroscopies with tunable optical and dynamic properties," Biomed. Opt. Express 9, 2068-2080 (2018).

51. A. Giusto et al., "Monitoring absorption changes in a layered diffusive medium by white-light time-resolved reflectance spectroscopy," IEEE Trans. Instrum. Meas. 59(7), 1925-1932 (2010).

52. G. Yu et al., "Time-dependent blood flow and oxygenation in human skeletal muscles measured with noninvasive near-infrared diffuse optical spectroscopies," J. Biomed. Opt. 10(2), 024027 (2005).

53. J. Selb et al., "Sensitivity of near-infrared spectroscopy and diffuse correlation spectroscopy to brain hemodynamics: simulations and experimental findings during hypercapnia," Neurophotonics 1(1), 015005 (2014).

54. A. Rajaram et al., "Simultaneous monitoring of cerebral perfusion and cytochrome c oxidase by combining broadband near-infrared spectroscopy and diffuse correlation spectroscopy," Biomed. Opt. Express $\mathbf{9}$, 2588-2603 (2018).

55. A. Dalla Mora et al., "Towards next-generation time-domain diffuse optics for extreme depth penetration and sensitivity," Biomed. Opt. Express 6(5), 1749-1760 (2015).

56. A. Pifferi et al., "New frontiers in time-domain diffuse optics, a review," J. Biomed. Opt. 21(9), 091310 (2016).

57. F. Martelli et al., "There's plenty of light at the bottom: statistics of photon penetration depth in random media," Sci. Rep. 6, 27057 (2016).

58. T. Binzoni et al., "Depth sensitivity of frequency domain optical measurements in diffusive media," Biomed. Opt. Express 8(6), 2990-3004 (2017).

59. S. Gunadi et al., "Spatial sensitivity and penetration depth of three cerebral oxygenation monitors," Biomed. Opt. Express 5, 2896-2912 (2014).

60. R. Re et al., "A compact time-resolved system for near infrared spectroscopy based on wavelength space multiplexing," Rev. Sci. Instrum. 81, 113101 (2010).

Martina Giovannella is a doctoral student at ICFO-The Institute of Photonic Sciences, Barcelona, Spain, in the Medical Optics Group. She received her master's degree in physics from the University of Pisa, Italy. Her research is focused on developing novel diffuse optical instrumentation, algorithms, and protocols for brain monitoring for preclinical and clinical studies.

Davide Contini received his MS degree in electronic engineering and his PhD in physics from Politecnico di Milano, Italy, in 2004 and 2007 , respectively. $\mathrm{He}$ is an associate professor in the Department of Physics, Politecnico di Milano. He has authored more than 150 papers in international peer-reviewed journals and conference proceedings. His research activity is focused on time-resolved spectroscopy of highly diffusive media for applications in biology and medicine.

Marco Pagliazzi is a PhD candidate in the Medical Optics Group at ICFO. He graduated in physics at the University of Pisa. The focus of his research is developing diffuse optical instrumentation, algorithms, and protocols, and applying them to the study of human physiology. 
Antonio Pifferi received his MS degree in nuclear engineering from Politecnico di Milano, in 1991, and his PhD in physics from Politecnico di Torino, Italy, in 1995. He is a full professor at the Department of Physics, Politecnico di Milano. His research is directed toward the development of laser techniques and instrumentation for diagnosis and the study of light propagation in diffusive media, with applications to optical biopsy, optical mammography, and functional brain imaging.

Lorenzo Spinelli received his MS degree and his $\mathrm{PhD}$ in physics from the University of Milan, Italy, in 1994 and 1999, respectively. Since 1999, he has been a postdoc in the Department of Physics at University of Milan. In 2001, he became a researcher for Italian Research National Council at Institute of Photonics and Nanotechnologies. His research interests are devoted to the study of photon migration in turbid media for optical biopsy and imaging.

Rainer Erdmann obtained its diploma degree in physics from Humboldt University Berlin, Germany, in 1999. Since 1996, he is the founder and CEO of PicoQuant GmbH. He is a lifetime member and fellow of SPIE (USA). He has authored more than 100 papers in international peer-reviewed journals and conference proceedings. His research activity is focused on time-resolved fluorescence spectroscopy and microscopy, single-molecule detection, and optical quantum technologies.

Roger Donat studied industrial engineering at the Universitat Politécnica de Catalunya, Barcelona, Spain. In his work at Loop, he has been involved in ideation and improvement of machines for a food sector company; creation of a new family of food packaging; business models; and development of products within the coffee sector; research in a new range of food products; conceptualization, design, and development of small appliances; and definition, development, manufacturing, testing, and management of prototypes.

Ignacio Rocchetti studied industrial engineering at the Universidad Tecnológica Nacional, Argentina, and obtained his master's degree in automation production and robotics at Universitat Politécnica de Catalunya, Barcelona. $\mathrm{He}$ is a partner at Loop. He has participated in more than 200 projects, in product and UX design and development to improve user experience for clients, such as Grifols, Biomet, Aglaris, and Nestle. In company and product strategy, he has managed projects for Indra, CAF, Orange, and Corporación Mondragon, among others.

Matthias Rehberger is a research associate and received his MS degree in physics from the University of Bonn, Germany, in 2013. He developed fiber-optical measurement systems and probes at the Fraunhofer Institute for Production Technology IPT. Since 2017, he is pursuing a $\mathrm{PhD}$ in the field of "Additive Manufacturing and Functional Layer" at the Fraunhofer Institute for Laser Technology ILT. His research activity is focused on laser-based additive manufacturing of highly integrated sensors.

Niels König is the head of the Department Production Metrology at Fraunhofer Institute for Production Technology IPT, Germany. He received his diploma in physics in 2007 from RWTH Aachen University, Germany. He has published more than 50 papers in international peer-reviewed journals and conference proceedings. His research is focused on fiber-optical sensors and applications in low-coherence interferometry and optical-coherence tomography, in particular.

Robert Schmitt is a full professor for production metrology and quality management at RWTH Aachen University. He received his MS degree in electrical engineering and his $\mathrm{PhD}$ in physics from RWTH Aachen. He has authored more than 490 international peerreviewed journals and conference proceedings. He is a member of the board of directors at Laboratory for Machine Tools and Production Engineering (WZL) and Fraunhofer Institute for Production Technology IPT, where he leads the production quality and metrology research area.

Alessandro Torricelli is a full professor in the Department of Physics, Politecnico di Milano. He received his MS degree in electronic engineering from Politecnico di Milano in 1994, and his $\mathrm{PhD}$ in physics from Politecnico di Torino in 1999. He has authored more than 100 papers in international peer-reviewed journals. His current research interests include photon migration in diffusive media, functional nearinfrared spectroscopy, and noninvasive diffuse spectroscopy with time domain systems.

Turgut Durduran was trained at the University of Pennsylvania, Philadelphia. In 2009, he moved to ICFO-The Institute of Photonic Sciences, where he leads the Medical Optics Group. His research interests revolve around the use of diffuse light to noninvasively probe tissue function. The Medical Optics Group develops new technologies and algorithms and routinely translates them to preclinical, clinical, and industrial applications.

Udo M. Weigel received his $\mathrm{PhD}$ in experimental physics from the University Heidelberg, Germany, in 2003, followed by a postdoctoral appointment at the Max-Planck-Institute for Nuclear Physics. From 2005 to 2009, he worked as an R\&D engineer in the semiconductor industry at the charged particle optics Department of ICT/Applied Materials Inc. In 2009, he joined ICFO's Medical Optics Group and became, in 2013, the CEO and co-founder of HemoPhotonics S.L. in Castelldefels (Barcelona), Spain. 